\title{
Optimización de la gestión del dolor postoperatorio basado en una nueva herramienta tecnológica. Mapa del dolor
}

\author{
D. Samper Bernal1 , P. Barroso Castaño², G. Roca Amatria ${ }^{3}$, M. Pérez Hinarejos ${ }^{4}$, M. M. Monerris Tabasco ${ }^{5}$ \\ L. García Eroles ${ }^{6}$ y R. López Alarcón?

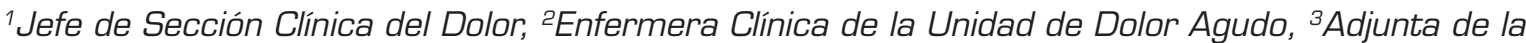 \\ Clínica del Dolor, ${ }^{4}$ Enfermera Supervisora y ${ }^{5}$ Adjunta de la Clínica del Dolor. Servicio de Anestesiología, \\ Reanimación y Terapéutica del dolor. Hospital Universitario Germans Trias i Pujol. Badalona. ${ }^{6}$ Gerente \\ de Sistemas de Información. Servicio Catalán de la Salud. 'Dirección de Organización y Sistemas de \\ Información. Gerencia Territorial Metropolitana Norte. Instituto Catalán de la Salud. España
}

\section{ABSTRACT}

Introduction: In 2005, the Ministry of Health, Social Services and Equality (MSSSI) promoted the Patient Safety Strategy of the National Health System (SNS), in collaboration with the Autonomous Communities, which integrated the contributions of health professionals and patients through their organizations.

One of the objectives of safe practices was the need for prevention and control of pain in adults and children, including management of the same in quality standards.

At the Germans Trias and Pujol University Hospital (HUGTIP) in 2016, the development of the indicators that were intended to be evaluated for better detection and therapeutic efficiency of acute in-hospital pain in any area ended with the design and creation of what we call as "pain map".

The objective was to design a tool that, through the clinical pain variable, could improve the visualization of pain and extract statistical data from the defined indicators for optimal pain management in a university hospital of the third level.

Material and method: Descriptive analysis of the tool we call pain map at the Germans Trias and Pujol University Hospital during 2016. The population was all patients admitted to hospital units where the pain registry was made through the GacelaCare ${ }^{\circledR}$ computerized medical record.

The main variable was the recording of pain intensity using the Visual Analogue Scale (VAS) or Numeric

Samper Bernal D, Barroso Castaño P, Roca Amatria G, Pérez Hinarejos M, Monerris Tabasco MM, García Eroles L y López Alarcón R. Optimización de la gestión del dolor postoperatorio basado en una nueva herramienta tecnológica. Mapa del dolor. Rev Soc Esp Dolor 2019;26(3):154-165.

\section{RESUMEN}

Introducción: En el año 2005 el Ministerio de Sanidad, Servicios Sociales e Igualdad (MSSSI) impulsó y promovió la Estrategia de Seguridad del Paciente del Sistema Nacional de Salud (SNS), en colaboración con las comunidades autónomas, que integraba las aportaciones de los profesionales sanitarios y de los pacientes a través de sus organizaciones.

Uno de los objetivos de las prácticas seguras era la necesidad de prevención y control del dolor en el adulto y en el niño, incluyéndose la gestión de este en los estándares de calidad.

En el Hospital Universitario Germans Trias i Pujol (HUGTIP), en el año 2016 se inició la elaboración de los indicadores que se querían evaluar para una mejor detección y eficiencia terapéutica del dolor agudo intrahospitalario de cualquier área y que finalizó con el diseño y creación de lo que denominamos "Mapa del dolor".

El objetivo fue diseñar una herramienta que, a través de la variable clínica dolor, permitiera mejorar la visualización de esta y extraer los datos estadísticos de los indicadores definidos para una óptima gestión del dolor dentro de un hospital universitario de tercer nivel.

Material y método: Análisis descriptivo de la herramienta que denominamos "Mapa del dolor" en el Hospital Universitario Germans Trias i Pujol (HUGTiP) durante el año 2016. Se incluyeron todos los pacientes ingresados en los que el registro de dolor se realizó a través de la historia clínica informatizada GacelaCare ${ }^{\circledR}$.

Recibido: 04-07-2018

Aceptado: 20-01-2019

Correspondencia: Daniel Samper Bernal dsamperb@gmail.com 
Verbal Scale (NVA) by nursing shift considering pain as the 5th constant. The tool creation procedure was carried out with the collaboration of the hospital information systems department together with members of the Pain Unit for the elaboration of the so-called pain map.

Results: The pain map is based on the previous action required by nursing which is the recording of the intensity of pain using the Visual Analogue Scale (VAS) or Numerical Verbal Scale (NVA) in turn considering pain as the 5th constant.

The map allows to visualize on the screen the registers of the pain intensity of all hospitalized patients simultaneously and in real time.

The pain map identifies with different colors as a traffic light of pain. In green color, the EVN values are between 0-3 (mild pain), orange EVN between 4 and 6 (moderate pain) and red color between 7 and 10 (intense pain). Likewise, it indicates with a purple band those patients operated on the previous day, facilitating pain control during the first 24 postoperative hours.

The statistical exploitation of computerized pain registers allows obtaining results of the quality indicators related to pain management defined by our hospital.

Discussion: The pain map is an innovative tool that allows the development of low-cost Acute Pain Units to be guaranteed with efficiency.

Key words: Pain, pain management, integrated and advanced information management systems, indicators of quality in health care, postoperative pain.
Considerando el dolor como 5. ${ }^{\text {a }}$ constante, la variable principal fue el registro de la intensidad del dolor mediante la escala visual analógica (EVA) o escala verbal numérica (EVN) en cada turno de enfermería. El procedimiento de creación de la herramienta para la elaboración del denominado "Mapa del dolor" se realizó con la colaboración del Departamento de Sistemas de Información del HUGTiP, junto con miembros de la Unidad del Dolor.

Resultados: El "Mapa del dolor" se fundamenta en el registro de la intensidad del dolor mediante la escala visual analógica (EVA) o escala verbal numérica (EVN) por turno de enfermería, acción previa imprescindible por parte de enfermería. Permite visualizar en la pantalla los registros de la intensidad del dolor de todos los pacientes hospitalizados de manera simultánea y en tiempo real.

El "Mapa del dolor" identifica los registros con distintos colores a modo de semáforo del dolor, en color verde marca los valores de EVN entre 1-3 (dolor leve), en color naranja EVN entre 4-6 (dolor moderado) y en color rojo EVN entre 7-10 (dolor intenso); asimismo señala con una franja de color morado aquellos pacientes operados el día anterior, facilitando el control del dolor durante las primeras 24 horas postoperatorias.

La explotación estadística de los registros informatizados de dolor permite obtener resultados de los indicadores relacionados con su gestión, previamente definidos por nuestro hospital.

Discusión: El "Mapa del dolor" es una herramienta innovadora que permite desarrollar con garantías la eficiencia de las unidades de dolor agudo de bajo coste.

Palabras clave: Dolor, manejo del dolor, sistemas integrados y avanzados de gestión de la información, indicadores de calidad de la atención de salud, dolor postoperatorio.

\section{INTRODUCCIÓN}

El Ministerio de Sanidad, Servicios Sociales e Igualdad (MSSSI), en colaboración con las comunidades autónomas, impulsó y promovió en el año 2005 la Estrategia de Seguridad del Paciente del Sistema Nacional de Salud (SNS), integrando las aportaciones de los profesionales sanitarios y de los pacientes a través de sus organizaciones $(1,2)$.

Los objetivos de dicha estrategia se fundamentaron en mejorar la cultura de la seguridad, la gestión del riesgo sanitario, la formación de los profesionales, la implementación de prácticas seguras, así como la implicación de los pacientes y los ciudadanos.

Los centros sanitarios deberían desarrollar sistemas eficaces para asegurar, a través de la Dirección Clínica, una asistencia segura y aprender lecciones de su propia práctica y de la de otros (3).

Uno de los objetivos de las prácticas seguras sería la necesidad de prevenir y controlar el dolor en el adulto y en el niño (4-6), incluyéndose la gestión de este en los estándares del "Achieving Improved Measurement" del Canadian Council on Health Services Accreditation (7).
En el Hospital Universitario Germans Trias i Pujol, desde el año 1990 hasta la actualidad, se han implementado paulatinamente estrategias y recursos para mejorar el control del dolor, que han ido desde la utilización de las bombas de analgesia controlada por el paciente (PCA) en los años 90 hasta la constitución del Comité del Dolor en el año 2009, con una amplia representación de miembros de servicios médicos, quirúrgicos y equipos de enfermería.

Este Comité ha sido y es el motor que, en menos de una década, ha consolidado la eficiencia en el control del dolor mediante la creación de la figura de la enfermera referente en dolor en cada unidad de hospitalización y servicios críticos, la protocolización de todo el dolor agudo de todos los servicios quirúrgicos y médicos y el análisis sistemático de la prevalencia del dolor intrahospitalario. Ha instaurado también la formación continuada de todo el personal de enfermería a través de un curso monográfico realizado de forma bianual (del que se han realizado 14 ediciones hasta la actualidad), siendo la última acción de dicho comité la incorporación de la enfermera clínica del dolor agudo a partir del año 2015. 
Por otro lado, las tecnologías de la información y la comunicación (TIC), dirigidas inicialmente a la gestión de los recursos hospitalarios, se han abierto actualmente a la monitorización y control de variables clínicas; por ello en el año 2016, utilizando sus recursos, se inició la elaboración de los indicadores de dolor agudo intrahospitalario.

El objetivo fue diseñar una herramienta que, a través de la variable clínica dolor, permitiera mejorar la visualización de esta, extrayendo asimismo los datos estadísticos de los indicadores definidos para una óptima gestión del dolor.

\section{MATERIAL Y MÉTODO}

Análisis descriptivo de la herramienta que denominamos "Mapa del dolor" en el Hospital Universitario Germans Trias i Pujol durante el año 2016.

La población estudiada fueron todos los pacientes ingresados, en los que el registro de dolor se realizó a través de la historia clínica informatizada GacelaCare ${ }^{\circledR}$.

La variable principal fue el registro de la intensidad del dolor mediante la Escala Visual Analógica (EVA) o Escala Verbal Numérica (EVN); considerando el dolor como 5. ${ }^{a}$ constante, dicho registro se realizó en cada turno de enfermería.

El procedimiento de creación de la herramienta se realizó con la colaboración del Departamento de Sistemas de Información y miembros del Comité del Dolor. Los objetivos fueron optimizar la visualización de los registros de intensidad del dolor y, a través de ello, definir los indicadores para una mejor detección y eficiencia terapéutica del dolor agudo intrahospitalario. Dicha herramienta se integró en el sistema "Business Objects" (BO), que obtiene los datos de GacelaCare ${ }^{\circledR}$.

La implantación de la nueva herramienta se realizó de manera simultánea a finales del año 2016 en 10 unidades de hospitalización de adultos y 1 de hospitalización pediátrica. A lo largo del año 2017 se incorporaron la Unidad de Trasplante Renal y la nueva unidad de hospitalización de pacientes con patología psiquiátrica, completando así el registro en todas las unidades de hospitalización de nuestro centro.

La visualización diaria de esta herramienta corre a cargo de la enfermera clínica de dolor agudo, detectando aquellos pacientes con registros altos de dolor, a fin de evaluar las causas y optimizar la pauta analgésica bajo tutela del facultativo asignado de la unidad.

Los registros con dolor intenso son evaluados juntamente con la enfermera del paciente, así como con la enfermera referente de dolor y la supervisora de cada una de las unidades de hospitalización.

\section{RESULTADOS}

El "Mapa del dolor" permite visualizar en pantalla los registros de la intensidad del dolor de todos los pacientes hospitalizados de manera simultánea y en tiempo real (Figura 1).

El "Mapa del dolor", diseñado de modo muy visual, identifica, con distintos colores a modo de semáforo, la intensidad del dolor. En color verde marca los valores de EVN entre 1-3 (dolor leve), en color naranja EVN entre 4-6 (dolor moderado) y en color rojo EVN entre 7-10 (dolor intenso). Asimismo, señala con una franja de color morado aquellos pacientes operados el día anterior, facilitando el control del dolor durante las primeras 24 horas postoperatorias (Figura 2).

Existen dos esquemas de visualización: uno proporciona información que permite detectar todos aquellos pacientes que tienen o han tenido dolor en las últimas 24 horas, y el otro durante todo el ingreso del paciente (Figura 3).

Esta información es la punta de lanza que inicia, desde la Unidad de Dolor Agudo, todas aquellas acciones dirigidas a aliviar, el máximo posible, el dolor de estos pacientes.

En la parte inferior del mapa del dolor se muestra la información general por planta de hospitalización y el total del hospital con descripción de camas libres, camas ocupadas, porcentajes de pacientes medidos con un registro de dolor por turno de enfermería, pacientes operados las últimas 24 horas, porcentaje de pacientes con dolor EVN > 3 y EVN media (Figura 4).

Se puede acceder a la pantalla de visualización de un paciente al pulsar sobre cualquier valor de la EVN del mapa del dolor, tras lo que aparecerá una pantalla con todos los registros de dolor desde que ingresó hasta el último registro tomado. Esto va acompañado de tablas y gráficos que ayudan a valorar si la analgesia instaurada ha sido eficaz (Figuras 5 y 6).

Además, el "Mapa del dolor" identifica si un paciente ha tenido un pico de dolor (EVN $\geq 7$ ) y si se ha administrado un rescate analgésico efectivo (a la hora y a las 2 horas de este pico de dolor), acción que demuestra si ha habido una intervención rápida y efectiva ante un dolor intenso (Figura 6).

Mediante la utilización de filtros, la herramienta permite la explotación de datos individualmente en cada servicio médico o quirúrgico y en cada planta de hospitalización, lo que facilita la valoración del control del dolor en cada uno de estos entornos (Figura 7).

A falta de indicadores de dolor agudo propuestos por las sociedades científicas, se definieron aquellos que consideramos imprescindibles para evaluar la eficiencia del dolor intrahospitalario, con especial énfasis en la eficacia de las acciones emprendidas ante un dolor intenso con el objetivo de identificar problemas e implementar medidas de mejora.

Entre los indicadores seleccionados, consideramos de gran interés aquellos que evaluaban el registro de la EVN, como mínimo, en cada turno de enfermería. Para conseguir los mejores resultados de estos indicadores se intensificó la formación a Enfermería sobre la importancia del correcto registro del dolor, así como de la revaloración del mismo tras un episodio de dolor intenso (EVA $\geq 7$ ).

Considerando que el dolor de mayor intensidad postquirúrgico no se presenta tan solo durante las primeras 24 horas, sino también entre las 48 y 72 horas, que es cuando el paciente postoperado intensifica la movilización, fisioterapia respiratoria, rehabilitación, etc., se establecieron indicadores durante todo este periodo. Los resultados de estos indicadores de dolor agudo postoperatorio nos han permitido revalorar los protocolos de pauta analgésica, sobretodo en aquellas cirugías fast-track donde es clave la optimización analgésica al alta precoz. 
Dolor ayer: de 400 camas ocupadas, 330 medidas $(82,5 \%)$ y 21 con dolor $(6,36 \%), 71$ en postoperatorio, 22 en primeras 24 horas postoperatorias
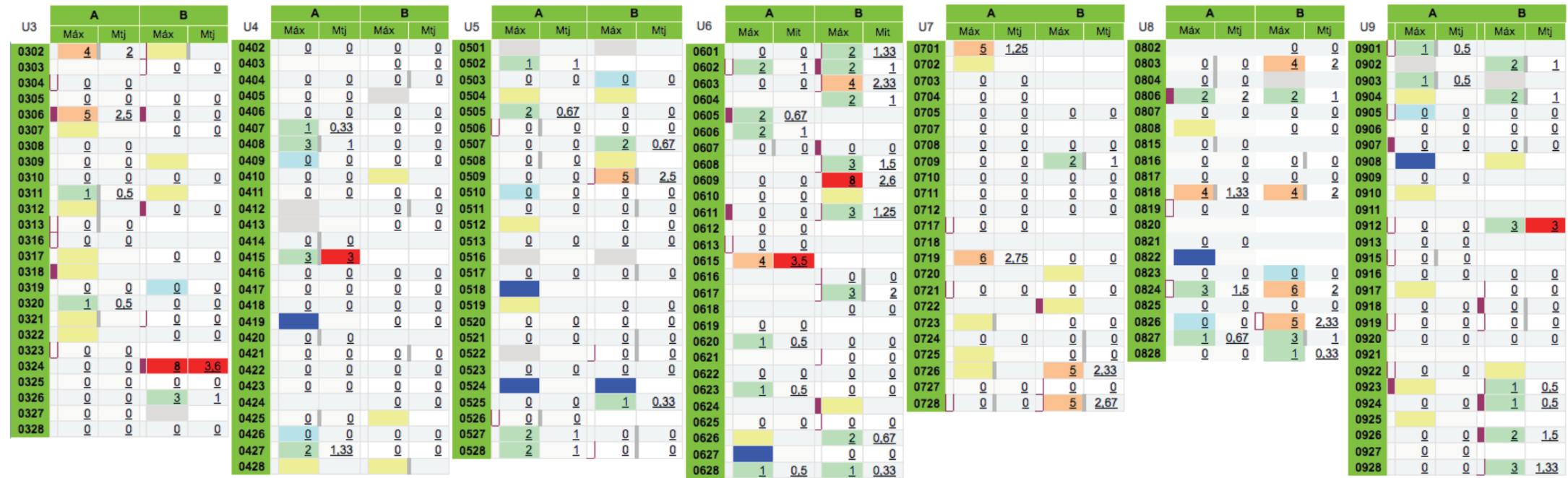

\begin{tabular}{|lc|lc|}
\multicolumn{2}{c|}{ Planta 3 General } & \multicolumn{2}{c|}{ Planta 4 General } \\
\hline Camas & 48 & Camas & 52 \\
\hline Ocupadas & 43 & Ocupadas & 50 \\
\hline Libres & 0 & Libres & 0 \\
\hline valorado el dolor & 39 & valorado el dolor & 34 \\
\hline$\%$ valorado dolor & $90,70 \%$ & \% valorado dolor & $87,18 \%$ \\
\hline Intervenidos ayer & 7 & Intervenidos ayer & 0 \\
\hline Dolor $>3$ & 5 & Dolor $>3$ & 4 \\
\hline$\%$ dolor $>3$ & $12,82 \%$ & \% dolor $>3$ & $8,70 \%$ \\
\hline EVAmedia & 0,57 & EVAmedia & 0,41 \\
\hline
\end{tabular}

\begin{tabular}{|lc|}
\hline \multicolumn{2}{|c|}{ Planta 5 General } \\
\hline Camas & 52 \\
\hline Ocupadas & 39 \\
\hline Libres & 0 \\
\hline valorado el dolor & 34 \\
\hline$\%$ valorado dolor & $87,18 \%$ \\
\hline Intervenidos ayer & 2 \\
\hline Dolor $>3$ & 2 \\
\hline$\%$ dolor $>3$ & $5,88 \%$ \\
\hline EVAmedia & 0,26 \\
\hline
\end{tabular}

\begin{tabular}{|lc|}
\hline \multicolumn{2}{|c|}{ Planta 6 General } \\
\hline Camas & 52 \\
\hline Ocupadas & 42 \\
\hline Libres & 0 \\
\hline valorado el dolor & 39 \\
\hline$\%$ valorado dolor & $92,86 \%$ \\
\hline Intervenidos ayer & 5 \\
\hline Dolor $>3$ & 4 \\
\hline$\%$ dolor $>3$ & $10,26 \%$ \\
\hline EVAmedia & 0,68 \\
\hline
\end{tabular}

\begin{tabular}{|lc|}
\multicolumn{2}{|c|}{ Planta 7 General } \\
\hline Camas & 39 \\
\hline Ocupadas & 31 \\
\hline Libres & 1 \\
\hline valorado el dolor & 27 \\
\hline$\%$ valorado dolor & $87,10 \%$ \\
\hline Intervenidos ayer & 2 \\
\hline Dolor $>3$ & 1 \\
\hline$\%$ dolor $>3$ & $3,70 \%$ \\
\hline EVAmedia & 0,22 \\
\hline
\end{tabular}

\begin{tabular}{|l|}
\multicolumn{1}{|l}{ Planta 8} \\
\hline Camas \\
\hline Ocupadas \\
\hline Libres \\
\hline valorado el dolor \\
\% valorado dol \\
Intervenidos ay \\
\hline Dolor $>3$ \\
\hline$\%$ dolor $>3$ \\
\hline EVAmedia \\
\hline 114 \\
\hline Postoperados \\
\hline
\end{tabular}

Planta 8 Ceneral valorado dolor $\quad 96,67 \%$

2

0,38

\begin{tabular}{|c|c|c|c|c|c|c|c|c|c|}
\hline 2 & 26 & 347 & 85 & 68 & 12 & 67 & 105 & 27 & 114 \\
\hline Dolor $\geq 7$ & Dolor $>3$ & Sin dolor & $\begin{array}{c}\text { Cama ocupada } \\
\text { dolor no } \\
\text { valorado }\end{array}$ & $\begin{array}{c}\text { Cama } \\
\text { bloqueada }\end{array}$ & $\begin{array}{c}\text { Cama } \\
\text { planificada }\end{array}$ & Cama libre & $\begin{array}{c}\text { Estancia > 15 } \\
\text { días }\end{array}$ & Operados ayer & Postoperados \\
\hline
\end{tabular}

Fig. 1. Visión general del "Mapa del dolor". 


\begin{tabular}{|c|c|c|c|c|c|c|c|c|c|c|c|c|c|c|c|c|c|c|c|}
\hline \multirow[b]{2}{*}{ U7 } & \multicolumn{2}{|c|}{ A } & \multicolumn{2}{|c|}{ B } & \multirow[b]{2}{*}{ U8 } & \multicolumn{2}{|l|}{ A } & \multicolumn{2}{|c|}{ B } & \multirow[b]{2}{*}{ U9 } & \multicolumn{2}{|c|}{ A } & \multicolumn{2}{|c|}{ B } & \multirow[b]{2}{*}{ U10 } & \multicolumn{2}{|c|}{ A } & \multicolumn{2}{|c|}{ B } \\
\hline & Máximo & Media & Máximo & Media & & Máximo & Media & Máximo & Media & & Máximo & Media & Máximo & Media & & Máximo & Media & Máximo & Media \\
\hline 0701 & $\underline{0}$ & $\underline{0}$ & & & 0802 & & & & & 0901 & $\underline{0}$ & $\underline{0}$ & $\underline{0}$ & $\underline{0}$ & 1007 & & & & \\
\hline 0702 & $\underline{0}$ & $\underline{\overline{0}}$ & & & 0803 & & & & & 0902 & $\underline{\overline{0}}$ & $\underline{0}$ & $\underline{\overline{3}}$ & $\underline{1, \overline{5}}$ & 1008 & $\underline{0}$ & $\underline{0}$ & & \\
\hline 0703 & $\underline{2}$ & $\underline{0.6 \overline{7}}$ & & & 0804 & & & & & 0903 & $\underline{\overline{0}}$ & $\underline{\overline{0}}$ & $\underline{\overline{0}}$ & $\underline{0}$ & 1009 & $\underline{\overline{0}}$ & $\underline{\overline{0}}$ & & \\
\hline 0704 & $\underline{2}$ & $\underline{0,25}$ & & & 0806 & & & & & 0904 & $\underline{0}$ & $\underline{0}$ & & & 1010 & $\underline{0}$ & $\underline{0}$ & $\underline{0}$ & $\underline{0}$ \\
\hline 0705 & $\underline{0}$ & $\underline{0}$ & $\underline{0}$ & $\underline{0}$ & 0807 & $\underline{0}$ & $\underline{0}$ & $\underline{0}$ & $\underline{0}$ & 0905 & $\underline{\overline{0}}$ & $\underline{0}$ & $\underline{0}$ & $\underline{0}$ & 1011 & & & $\underline{0}$ & $\underline{0}$ \\
\hline 0707 & $\underline{0}$ & $\underline{0}$ & & & 0808 & $\underline{0}$ & $\underline{0}$ & $\underline{0}$ & $\underline{0}$ & 0906 & $\underline{0}$ & $\underline{0}$ & $\underline{0}$ & $\underline{0}$ & 1015 & & & $\underline{2}$ & 1 \\
\hline 0708 & $\underline{0}$ & $\underline{0}$ & $\underline{0}$ & $\underline{0}$ & 0815 & $\underline{0}$ & $\underline{0}$ & & & 0907 & $\underline{0}$ & $\underline{0}$ & $\underline{2}$ & 1 & 1016 & $\underline{2}$ & $\underline{2}$ & $\underline{0}$ & $\underline{0}$ \\
\hline 0709 & $\underline{3}$ & $\underline{2}$ & $\underline{0}$ & $\underline{0}$ & 0816 & $\underline{0}$ & $\underline{0}$ & 1 & $\underline{0.67}$ & 0908 & $\underline{0}$ & $\underline{0}$ & $\underline{0}$ & $\underline{0}$ & 1017 & $\underline{0}$ & $\underline{0}$ & $\underline{0}$ & $\underline{0}$ \\
\hline 0710 & $\underline{2}$ & $\underline{0,67}$ & $\underline{2}$ & $\underline{0,67}$ & 0817 & $\underline{0}$ & $\underline{0}$ & $\underline{0}$ & $\underline{0}$ & 0909 & & & $\underline{0}$ & $\underline{0}$ & 1018 & $\underline{0}$ & $\underline{0}$ & $\underline{7}$ & $\underline{2,25}$ \\
\hline 0711 & & & $\underline{0}$ & $\underline{0}$ & 0818 & $\underline{0}$ & $\underline{0}$ & $\underline{0}$ & $\underline{0}$ & 0910 & $\underline{0}$ & $\underline{0}$ & $\underline{0}$ & $\underline{0}$ & 1019 & $\underline{0}$ & $\underline{0}$ & $\underline{2}$ & $\underline{0,67}$ \\
\hline 0712 & $\underline{2}$ & $\underline{0.67}$ & $\underline{2}$ & $\underline{0.6 \overline{7}}$ & 0819 & $\underline{0}$ & $\underline{0}$ & & & 0911 & $\underline{\overline{0}}$ & $\underline{\overline{0}}$ & $\underline{\overline{0}}$ & $\underline{\overline{0}}$ & 1020 & & & $\underline{0}$ & $\underline{0}$ \\
\hline 0717 & $\underline{0}$ & $\underline{0}$ & & & 0820 & 1 & $\underline{0,14}$ & & & 0912 & & & & & 1021 & $\underline{0}$ & $\underline{0}$ & $\underline{0}$ & $\underline{0}$ \\
\hline 0718 & $\underline{0}$ & $\underline{0}$ & & & 0821 & $\underline{0}$ & $\underline{0}$ & & & 0913 & $\underline{0}$ & $\underline{0}$ & & & 1022 & $\underline{0}$ & $\underline{0}$ & $\underline{0}$ & $\underline{0}$ \\
\hline 0719 & $\underline{0}$ & $\underline{0}$ & $\underline{0}$ & $\underline{0}$ & 0822 & $\underline{0}$ & $\underline{0}$ & & & 0915 & 1 & $\underline{0,33}$ & & & 1023 & $\underline{0}$ & $\underline{0}$ & $\underline{0}$ & $\underline{0}$ \\
\hline 0720 & $\underline{0}$ & $\underline{0}$ & $\underline{1}$ & $\underline{0,3 \overline{3}}$ & 0823 & $\underline{0}$ & $\underline{0}$ & $\underline{0}$ & $\underline{0}$ & 0916 & $\underline{1}$ & $\underline{0,33}$ & 1 & $\underline{0.5}$ & 1024 & & & & \\
\hline 0721 & $\underline{0}$ & $\underline{0}$ & $\underline{0}$ & $\underline{0}$ & 0824 & $\underline{0}$ & $\underline{0}$ & $\underline{0}$ & $\underline{0}$ & 0917 & $\underline{\bar{z}}$ & $\underline{3,25}$ & $\underline{4}$ & $\underline{1,25}$ & & & & & \\
\hline 0722 & $\underline{0}$ & $\underline{0}$ & $\underline{7}$ & $\underline{2.33}$ & 0825 & $\underline{0}$ & $\underline{0}$ & $\underline{0}$ & $\underline{0}$ & 0918 & 1 & $\underline{0,33}$ & $\underline{4}$ & $\underline{1.25}$ & & & & & \\
\hline 0723 & $\overline{5}$ & 3,33 & $\underline{2}$ & $\overline{1,33}$ & 0826 & $\underline{0}$ & $\underline{0}$ & $\underline{2}$ & $\underline{0,67}$ & 0919 & $\overline{1}$ & $\underline{0,33}$ & $\underline{4}$ & $\overline{1,67}$ & & & & & \\
\hline 0724 & 1 & $\underline{0,5}$ & $\underline{0}$ & $\underline{0}$ & 0827 & $\underline{0}$ & $\underline{0}$ & $\underline{4}$ & 1,25 & 0920 & 1 & $\overline{0,33}$ & & & & & & & \\
\hline 0725 & $\underline{0}$ & $\underline{0}$ & $\underline{0}$ & $\underline{0}$ & 0828 & $\underline{2}$ & $\underline{0.5}$ & $\underline{0}$ & $\underline{0}$ & 0921 & $\underline{4}$ & $\underline{1,33}$ & $\underline{0}$ & $\underline{0}$ & & & & & \\
\hline 0726 & $\underline{\overline{0}}$ & $\underline{0}$ & $\underline{\overline{0}}$ & $\underline{0}$ & & & & & & 0922 & $\underline{0}$ & $\underline{0}$ & $\overline{4}$ & $1,2 \overline{5}$ & & & & & \\
\hline 0727 & $\underline{0}$ & $\underline{0}$ & $\underline{0}$ & $\underline{0}$ & & & & & & 0923 & $\underline{4}$ & 3,25 & $\underline{5}$ & $\underline{2,5}$ & & & & & \\
\hline 0728 & $\underline{0}$ & $\underline{0}$ & $\underline{3}$ & $\underline{2.67}$ & & & & & & 0924 & $\underline{0}$ & $\underline{0}$ & & & & & & & \\
\hline & & & & & & & & & & 0925 & $\underline{0}$ & $\underline{0}$ & $\underline{0}$ & $\underline{0}$ & & & & & \\
\hline & & & & & & & & & & 0926 & $\underline{2}$ & 1 & 1 & $\underline{0,3 \overline{3}}$ & & & & & \\
\hline & & & & & & & & & & 0927 & $\underline{3}$ & $\underline{0,75}$ & $\underline{2}$ & $\underline{0,67}$ & & & & & \\
\hline & & & & & & & & & & 0928 & $\underline{0}$ & $\underline{0}$ & $\underline{2}$ & $\underline{0,67}$ & & & & & \\
\hline
\end{tabular}

Semáforo del dolor. EVN máximo: O (no dolor) color blanco; entre 1-3 (dolor leve) color verde; entre 4-6 (dolor moderado) color naranja; entre 7-10 (dolor intenso) color rojo. EVN media: media > 3 color rojo. Barras de color morado: pacientes operados el día anterior. doble línea morada: pacientes operados durante su ingreso. 
Y) Mapa de Dolor: información correspondiente a jueves, 14 de junio de 2018

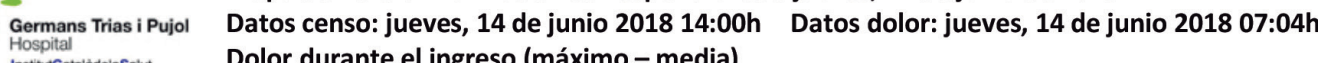

Insthinçatalaldelassahit Dolor durante el ingreso (máximo - media)

Dolor durante el ingreso: de 458 camas ocupadas, 387 medidas $(84,5 \%)$ y 121 con dolor $(31,27 \%), 114$ en postoperatorio, 27 en primeras 24 horas postoperatorias
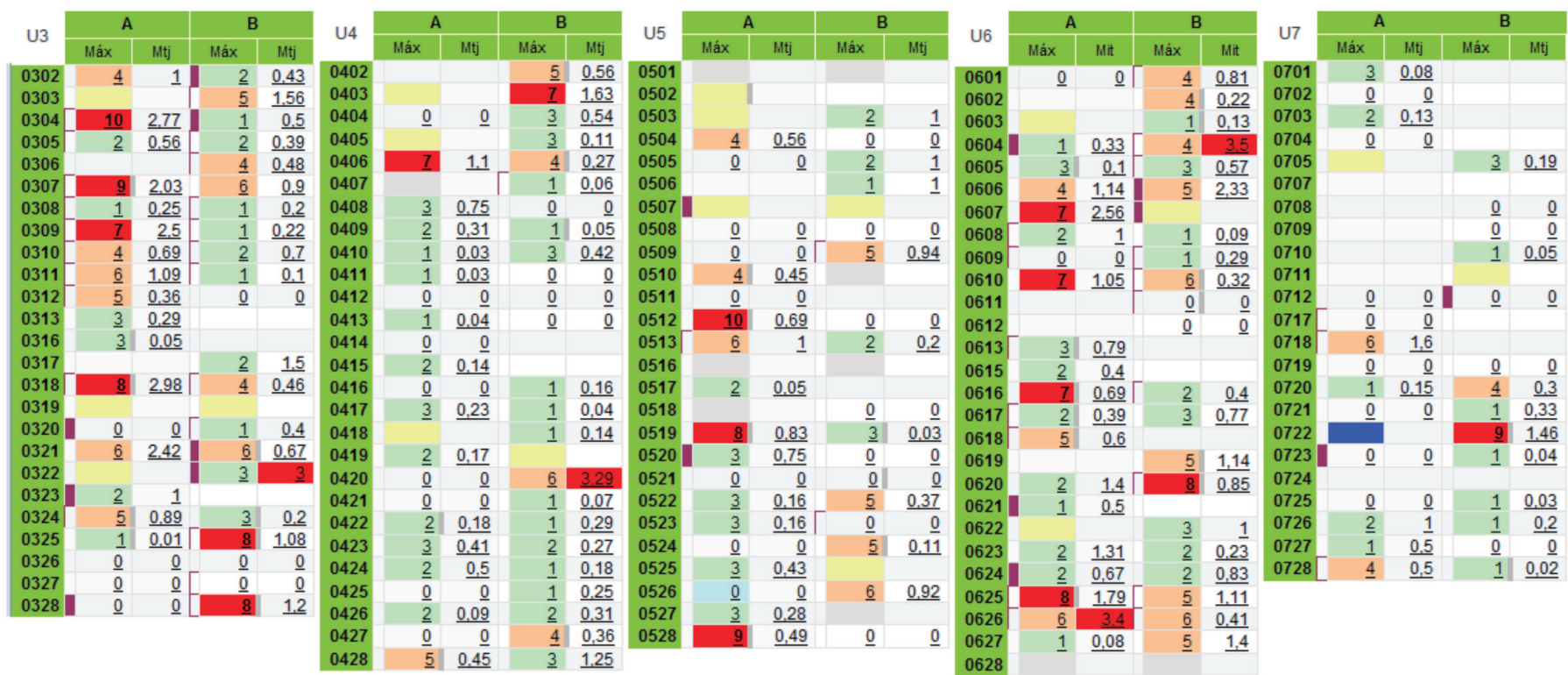

\begin{tabular}{l|l|l|lll}
0606 & $\underline{4}$ & $\frac{1,14}{2.56}$ & $\underline{5}$ & $\underline{2,33}$ & 0707 \\
0708
\end{tabular}

$\begin{array}{lllllll}0607 & \frac{7}{2} & \frac{2.56}{1} & 1 & 0.09 & 0709 \\ 0608 & \frac{2}{1} & 1 & 0708\end{array}$

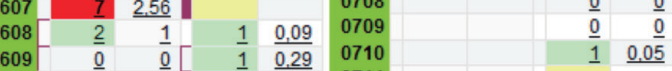

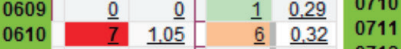

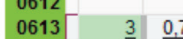

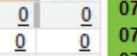

$\begin{array}{llllllllllll}0712 & 0 & 0 & 0 & 0 & 0819 & \frac{3}{0} & \frac{0.56}{0} & \frac{2}{3} & \frac{0.18}{0.22}\end{array}$

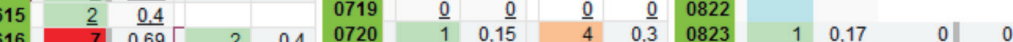

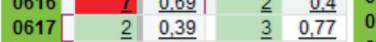

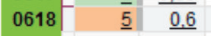

0619

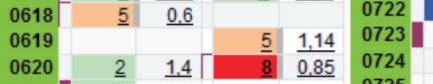

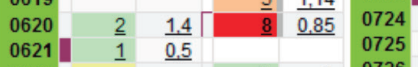

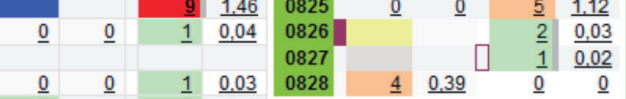

$\begin{array}{llllllllll}0.31 & \frac{3}{2} & \frac{1}{2} & 0726 & \frac{2}{1} & \frac{1}{5} & \frac{1}{0} & \frac{0,2}{0}\end{array}$

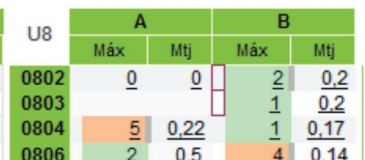

Dolor aye

Dolor durante ingreso

Fig. 3. "Mapa del dolor" durante el ingreso. 


\begin{tabular}{|c|c|c|c|c|c|c|c|c|c|c|c|}
\hline \multicolumn{2}{|c|}{ Planta 3 General } & \multicolumn{2}{|c|}{ Planta 4 General } & \multicolumn{2}{|c|}{ Planta 5 General } & \multicolumn{2}{|c|}{ Planta 6 General } & \multicolumn{2}{|c|}{ Planta 7 General } & \multicolumn{2}{|c|}{ Planta 8 General } \\
\hline Camas & 48 & Camas & 52 & Camas & 52 & Camas & 52 & Camas & 39 & Camas & 35 \\
\hline Ocupadas & 43 & Ocupadas & 50 & Ocupadas & 39 & Ocupadas & 42 & Ocupadas & 31 & Ocupadas & 30 \\
\hline Libres & 0 & Libres & 0 & Libres & 0 & Libres & 0 & Libres & 1 & Libres & 0 \\
\hline$\%$ valorado dolor & $90,70 \%$ & $\%$ valorado dolor & $87,18 \%$ & $\%$ valorado dolor & $87,18 \%$ & $\%$ valorado dolor & $92,86 \%$ & $\%$ valorado dolor & $87,10 \%$ & $\%$ valorado dolor & $96,67 \%$ \\
\hline Intervenidos ayer & 7 & Intervenidos ayer & 0 & Intervenidos ayer & 2 & Intervenidos ayer & 5 & Intervenidos ayer & 2 & Intervenidos ayer & 2 \\
\hline Dolor $>3$ & 5 & Dolor $>3$ & 4 & Dolor $>3$ & 2 & Dolor $>3$ & 4 & Dolor $>3$ & 1 & Dolor $>3$ & 3 \\
\hline
\end{tabular}

\begin{tabular}{|lc|}
\hline \multicolumn{2}{|c|}{ Planta 9 General } \\
\hline Camas & 52 \\
\hline Ocupadas & 41 \\
\hline Libres & 0 \\
\hline valorado el dolor & 38 \\
\hline \% valorado dolor & $92,68 \%$ \\
\hline Intervenidos ayer & 7 \\
\hline Dolor $>3$ & 2 \\
\hline$\%$ dolor $>3$ & $5,26 \%$ \\
\hline EVA media & 0,34 \\
\hline
\end{tabular}

\begin{tabular}{|lc|}
\hline \multicolumn{2}{|c|}{ Todo el hospital } \\
\hline Camas & 608 \\
\hline Ocupadas & 425 \\
\hline Libres & 61 \\
\hline valorado el dolor & 356 \\
\hline$\%$ valorado dolor & $83,76 \%$ \\
\hline Intervenidos ayer & 27 \\
\hline Dolor $>3$ & 26 \\
\hline$\%$ dolor $>3$ & $7,30 \%$ \\
\hline EVAmedia & 0,42 \\
\hline
\end{tabular}

\begin{tabular}{|c|c|c|c|c|c|c|c|c|c|}
\hline 2 & 26 & 347 & 85 & 68 & 12 & 67 & 105 & 27 & 114 \\
\hline Dolor $\geq 7$ & Dolor $>3$ & Sin dolor & $\begin{array}{c}\text { Cama ocupada } \\
\text { dolor no } \\
\text { valorado }\end{array}$ & $\begin{array}{c}\text { Cama } \\
\text { bloqueada }\end{array}$ & $\begin{array}{c}\text { Cama } \\
\text { planificada }\end{array}$ & Cama libre & $\begin{array}{c}\text { Estancia >15 } \\
\text { dias }\end{array}$ & Operados aver & Postoperados \\
\hline
\end{tabular}

Fig. 4. Datos de las plantas de hospitalización y de todo el hospital. 


\begin{tabular}{|c|c|c|c|c|c|c|c|c|c|c|c|c|c|c|c|c|c|c|c|}
\hline \multirow[b]{2}{*}{ U7 } & \multicolumn{2}{|c|}{ A } & \multicolumn{2}{|c|}{ B } & \multirow[b]{2}{*}{ U8 } & \multicolumn{2}{|c|}{ A } & \multicolumn{2}{|c|}{ B } & \multirow[b]{2}{*}{ U9 } & \multicolumn{2}{|c|}{ A } & \multicolumn{2}{|c|}{ B } & \multirow[b]{2}{*}{ U10 } & \multicolumn{2}{|c|}{ A } & \multicolumn{2}{|c|}{ B } \\
\hline & Máximo & Media & Máximo & Media & & Máximo & Media & Máximo & Media & & Máximo & Media & Máximo & Media & & Máximo & Media & Máximo & Media \\
\hline 0701 & $\underline{0}$ & $\underline{0}$ & & & 0802 & & & & & 0901 & $\underline{0}$ & $\underline{0}$ & $\underline{0}$ & $\underline{0}$ & 1007 & & & & \\
\hline 0702 & $\underline{0}$ & $\underline{0}$ & & & 0803 & & & & & 0902 & $\underline{0}$ & $\underline{0}$ & $\underline{3}$ & $\underline{1,5}$ & 1008 & $\underline{0}$ & $\underline{0}$ & & \\
\hline 0703 & $\underline{2}$ & $\underline{0,67}$ & & & 0804 & & & & & 0903 & $\underline{0}$ & $\underline{0}$ & $\underline{0}$ & $\underline{0}$ & 1009 & $\underline{0}$ & $\underline{0}$ & & \\
\hline 0704 & $\underline{2}$ & $\underline{0,25}$ & & & 0806 & & & & & 0904 & $\underline{0}$ & $\underline{0}$ & & & 1010 & $\underline{0}$ & $\underline{0}$ & $\underline{0}$ & $\underline{0}$ \\
\hline 0705 & $\underline{0}$ & $\underline{0}$ & $\underline{0}$ & $\underline{0}$ & 0807 & $\underline{0}$ & $\underline{0}$ & $\underline{0}$ & $\underline{0}$ & 0905 & $\underline{0}$ & $\underline{0}$ & $\underline{0}$ & $\underline{0}$ & 1011 & & & $\underline{0}$ & $\underline{0}$ \\
\hline 0707 & $\underline{0}$ & $\underline{0}$ & & & 0808 & $\underline{0}$ & $\underline{0}$ & $\underline{0}$ & $\underline{0}$ & 0906 & $\underline{0}$ & $\underline{0}$ & $\underline{0}$ & $\underline{0}$ & 1015 & & & $\underline{2}$ & 1 \\
\hline 0708 & $\underline{0}$ & $\underline{0}$ & $\underline{0}$ & $\underline{0}$ & 0815 & $\underline{0}$ & $\underline{0}$ & & & 0907 & $\underline{0}$ & $\underline{0}$ & $\underline{2}$ & 1 & 1016 & $\underline{2}$ & $\underline{2}$ & $\underline{0}$ & $\underline{0}$ \\
\hline 0709 & $\underline{3}$ & $\underline{2}$ & $\underline{0}$ & $\underline{0}$ & 0816 & $\underline{0}$ & $\underline{0}$. & 1 & $\underline{0,67}$ & 0908 & $\underline{0}$ & $\underline{0}$ & $\underline{0}$ & $\underline{0}$ & 1017 & $\underline{0}$ & $\underline{0}$ & $\underline{\overline{0}}$ & $\underline{0}$ \\
\hline 0710 & $\underline{2}$ & $\underline{0,67}$ & $\underline{2}$ & $\underline{0,67}$ & 0817 & $\underline{0}$ & $\underline{0}$ & $\underline{0}$ & $\underline{0}$ & 0909 & & & $\underline{0}$ & $\underline{0}$ & 1018 & $\underline{0}$ & $\underline{0}$ & $\underline{7}$ & $\underline{2,25}$ \\
\hline 0711 & & & $\underline{0}$ & $\underline{0}$ & 0818 & $\underline{0}$ & $\underline{0}$ & $\underline{0}$ & $\underline{0}$ & 0910 & $\underline{0}$ & $\underline{0}$ & $\underline{0}$ & $\underline{0}$ & 1019 & $\underline{0}$ & $\underline{0}$ & $\underline{2}$ & $\overline{0,67}$ \\
\hline 0712 & $\underline{2}$ & $\underline{0,67}$ & $\underline{2}$ & $\underline{0.67}$ & 0819 & $\underline{0}$ & $\underline{0}$ & & & 0911 & $\underline{0}$ & $\underline{0}$ & $\underline{0}$ & $\underline{0}$ & 1020 & & & $\underline{0}$ & $\underline{0}$ \\
\hline 0717 & $\overline{0}$ & $\underline{0}$ & & & 0820 & $\overline{1}$ & $\underline{0,1 \overline{4}}$ & & & 0912 & & & & & 1021 & $\underline{0}$ & $\underline{0}$ & $\underline{0}$ & $\overline{0}$ \\
\hline 0718 & $\underline{0}$ & $\underline{0}$ & & & 0821 & $\underline{0}$ & $\underline{0}$ & & & 0913 & $\underline{0}$ & $\underline{0}$ & & & 1022 & $\underline{0}$ & $\underline{0}$ & $\underline{0}$ & $\underline{0}$ \\
\hline 0719 & $\underline{0}$ & $\underline{0}$ & $\underline{0}$ & $\underline{0}$ & 0822 & $\underline{0}$ & $\underline{0}$ & & & 0915 & 1 & $\underline{0,33}$ & & & 1023 & $\underline{0}$ & $\underline{0}$ & $\underline{0}$ & $\underline{0}$ \\
\hline 0720 & $\underline{0}$ & $\underline{\overline{0}}$ & 1 & $\underline{0,3 \overline{3}}$ & 0823 & $\underline{0}$ & $\underline{0}$ & $\underline{0}$ & $\underline{0}$ & 0916 & $\overline{1}$ & $\underline{\underline{0,33}}$ & 1 & $\underline{0.5}$ & 1024 & & & & \\
\hline 0721 & $\underline{0}$ & $\underline{0}$ & $\underline{0}$ & $\underline{0}$ & 0824 & $\underline{0}$ & $\underline{0}$ & $\underline{0}$ & $\underline{0}$ & 0917 & $\underline{7}$ & 3,25 & $\underline{4}$ & $\underline{1,25}$ & & & & & \\
\hline 0722 & $\underline{0}$ & $\underline{0}$ & $\underline{7}$ & $\underline{2,33}$ & 0825 & $\underline{\overline{0}}$ & $\underline{0}$ & $\underline{\overline{0}}$ & $\underline{0}$ & 0918 & 1 & $\underline{0,33}$ & $\underline{4}$ & $\overline{1,25}$ & & & & & \\
\hline 0723 & $\underline{5}$ & 3.33 & $\underline{2}$ & $\overline{1,33}$ & 0826 & $\underline{0}$ & $\underline{0}$. & $\underline{2}$ & $\underline{0,67}$ & 0919 & 1 & $\underline{0.33}$ & $\underline{4}$ & $\overline{1.67}$ & & & & & \\
\hline 0724 & 1 & $\underline{0,5}$ & $\underline{0}$ & $\underline{0}$ & 0827 & $\underline{0}$ & $\underline{0}$ & $\underline{4}$ & 1,25 & 0920 & 1 & $\underline{0,33}$ & & & & & & & \\
\hline 0725 & $\underline{0}$ & $\underline{0}$ & $\underline{0}$ & $\underline{0}$ & 0828 & $\underline{2}$ & $\underline{0.5}$ & $\underline{0}$ & $\underline{0}$ & 0921 & $\underline{4}$ & $\underline{1,33}$ & $\underline{0}$ & $\underline{0}$ & & & & & \\
\hline 0726 & $\underline{0}$ & $\underline{0}$ & $\underline{0}$ & $\underline{0}$ & & & & & & 0922 & $\underline{0}$ & $\underline{0}$ & 4 & 1,25 & & & & & \\
\hline 0727 & $\underline{0}$ & $\underline{0}$ & $\underline{0}$ & $\overline{0}$ & & & & & & 0923 & $\underline{4}$ & 3,25 & $\overline{5}$ & $\overline{2,5}$ & & & & & \\
\hline 0728 & $\underline{0}$ & $\underline{0}$ & $\underline{3}$ & $\underline{2.67}$ & & & & & & 0924 & $\underline{0}$ & $\underline{0}$ & & & & & & & \\
\hline & & & & & & & & & & 0925 & $\underline{0}$ & $\underline{0}$ & $\underline{0}$ & $\underline{0}$ & & & & & \\
\hline & & & & & & & & & & 0926 & $\underline{2}$ & $\underline{1}$ & 1 & $\underline{0,3 \overline{3}}$ & & & & & \\
\hline & & & & & & & & & & 0927 & $\underline{3}$ & $\underline{0,75}$ & $\underline{2}$ & $\underline{0,67}$ & & & & & \\
\hline & & & & & & & & & & 0928 & $\underline{0}$ & $\underline{0}$ & $\underline{2}$ & $\underline{0,67}$ & & & & & \\
\hline
\end{tabular}

Al pulsar sobre cualquier valor de dolor de un paciente nos aparece una pantalla con todos los registros de dolor del paciente desde el día que ingresó hasta el día de hoy. Estos datos van acompañados de tablas y gráficos que ayudan a valorar si la analgesia instaurada ha sido eficaz.

Fig. 5. Visión específica. 


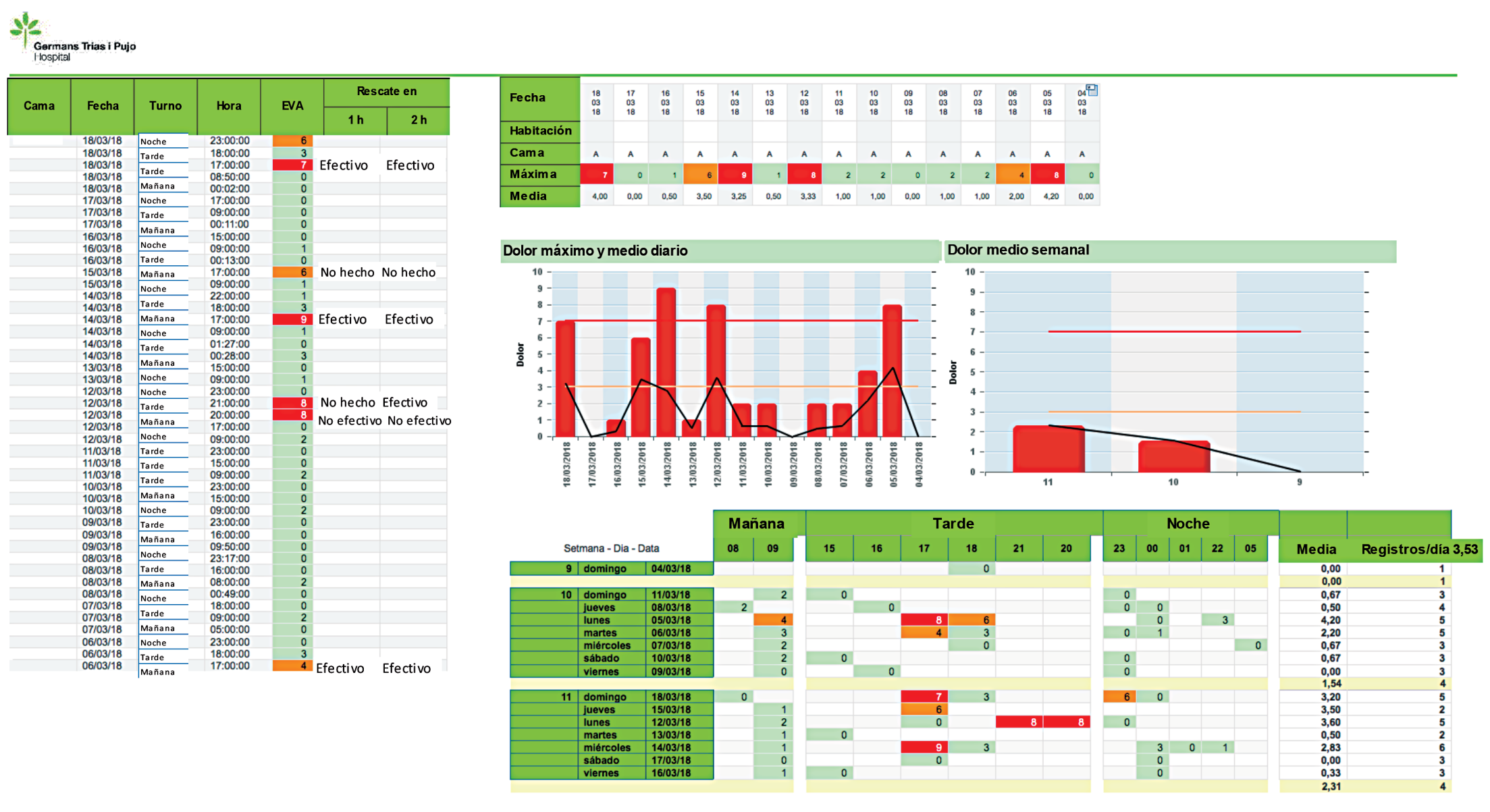

Fig. 6. "Mapa del dolor" de un paciente. 


\begin{tabular}{l} 
Qermans Trias i Pujol \\
Hospital \\
Institut Català de la Salut \\
Quirúrgicos \\
\hline Cirugía vascular \\
\hline Cirugía cardíaca \\
\hline Cirugía general y digestiva \\
\hline Cirugía ortopédica y traumatológica \\
\hline Cirugía plástica \\
\hline Cirugía torácica \\
\hline Neurocirugía \\
\hline ORL \\
\hline Urología \\
\hline
\end{tabular}

\section{Mapa de dolor Hospitalario}

\section{Servicios}

\begin{tabular}{l} 
Médicos \\
\hline Medicina interna \\
\hline Cardiología \\
\hline Neumología \\
\hline Neurología \\
\hline Digestología y hepatología \\
\hline Geriatría \\
\hline Nefrología \\
\hline Rehabilitación \\
\hline Reumatología \\
\hline Unidad orto-geriatría \\
\hline
\end{tabular}

\section{Médico-quirúrgico \\ Ginecología y obstetricia}

\begin{tabular}{l} 
Pediatría \\
Cirugía pediátrica \\
\hline Pediatría \\
\hline
\end{tabular}

Germans Trias i Pujol

Hospital

Institut Català de la Salut
Mapa de Dolor: información correspondiente a martes, 30 enero de 2018 Datos censo: martes, 30 enero 2018 16:00h

Dolor ayer (máximo - media)
Datos dolor: martes, 30 enero 2018 07:03h

Cirugía General y Digestiva

Dolor ayer: 39 camas ocupadas, 33 medidas $(84,6 \%$ ) y 1 con dolor $(3,03 \%)$

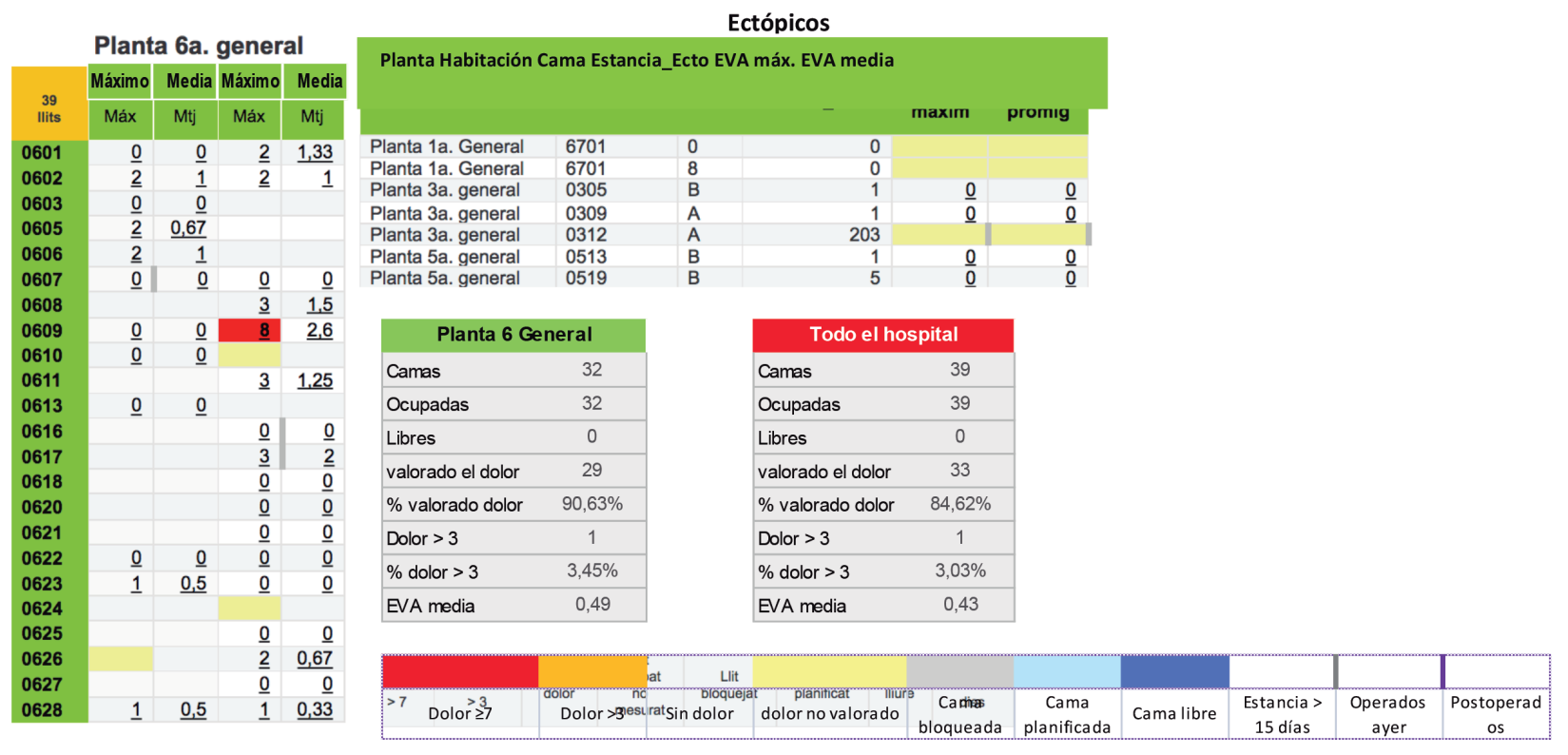

Fig. 7. Mapa de dolor hospitalario por servicios. 
La explotación estadística de estos datos permite obtener tablas comparativas de dichos indicadores de calidad, lo que permite la elaboración mensual de informes que se envían a todos los responsables de los servicios y unidades de hospitalización (Tablas I, II y III). Esta acción permite una gestión transversal del control del dolor hospitalario.

TABLA I

INDICADORES DE DOLOR: REGISTROS DE DOLOR

$\%$ pacientes con registros de la valoración del dolor

$\%$ registros con dolor de moderado a intenso (EVA > 3)

$\%$ registros con dolor intenso (EVA $\geq 7$ )

$\%$ registros con revaloración del pico de dolor de moderado a intenso (EVA > 3) y de dolor intenso (EVA $\geq 7$ ) en 1 hora

$\%$ registros con dolor leve (EVA $\leq 3$ ) después de 1 hora del pico de dolor intenso

\% registros con revaloración del pico de dolor de moderado a intenso (EVA > 3) y de dolor intenso (EVA $\geq 7$ ) en 2 horas

$\%$ registros con dolor leve (EVA $\leq 3$ ) después de 2 horas del pico de dolor intenso

TABLA II

INDICADORES DOLOR: POR PACIENTES

EVA media

$\%$ pacientes con dolor leve (EVA 1-3)

$\%$ pacientes con dolor moderado (EVA 4-6)

$\%$ pacientes con dolor intenso (EVA 7-10)

$\%$ pacientes con dolor de moderado a intenso (EVA $>3$ )

$\%$ pacientes con dolor intenso (EVA $\geq 7$ )

TABLA III

INDICADORES DOLOR: POR PACIENTES POSTQUIRÚRGICOS

Primeras 24, 48 y 72 horas postoperatorias (PO)
EVA media primeras 24, 48 y 72 h PO
\% pacientes con dolor de moderado a intenso
(EVA > 3) las primeras 24, 48 y 72 h PO
\% pacientes con dolor intenso (EVA $\geq 7$ ) las
primeras 24, 48 y 72 h PO
$\%$ pacientes con dolor leve [EVA $\leq 3$ ] después de
1 hora del pico de dolor intenso
$\%$ pacientes con dolor leve [EVA $\leq 3$ ] después de
2 horas del pico de dolor intenso

\section{DISCUSIÓN}

Las tecnologías de la información y la comunicación (TIC) en sanidad son herramientas clave para mejorar la calidad de la asistencia sanitaria y la salud de la ciudadanía, facilitar el trabajo de los profesionales y hacer que los centros sanitarios sean más eficientes [8].

El "Mapa del dolor" permite visualizar en tiempo real los valores de EVN de todos los pacientes hospitalizados (9), convirtiéndose en una herramienta de gestión fundamental para la Unidad de Dolor Agudo.

La formación específica en el ámbito del dolor, especialmente a Enfermería pero también a facultativos, es una acción imprescindible para el control del dolor. La designación de la enfermera clínica de dolor, junto al desarrollo de las TIC, permite la optimización de dicho control garantizando la eficacia, seguridad y calidad del cuidado.

El potencial de este instrumento de medida ha marcado muchas de las estrategias posteriores: utilizar correctamente las diferentes escalas de valoración del dolor, mejorar el grado de cumplimiento de valoración y registro en la historia clínica, corregir las prácticas incorrectas mediante la implicación y formación de los profesionales sanitarios, aplicar las diferentes medidas terapéuticas (farmacológicas y no farmacológicas), mejorar la valoración de la eficacia del tratamiento aplicado, optimizar la actuación ante un dolor intenso, informar a los pacientes sobre el manejo del dolor y, por último, minimizar el riesgo de errores en la administración de analgesia.

Creemos que conseguir la excelencia en el control del dolor agudo será una realidad cuando esté controlado el dolor en movimiento (tos, movimientos intestinales, respiración profunda, movimientos articulares, deambulación, etc.), no obstante debemos ser realistas y esto no se conseguirá si antes no somos capaces de controlar el dolor en reposo.

Los indicadores de dolor deben, pues, contemplar tanto el dolor en reposo como en movimiento.

Esta herramienta de control pretende ser el paso inicial para dar respuesta a todos los pacientes hospitalizados con dolor desde el reposo a la funcionalidad completa.

En definitiva, todas estas estrategias tienen como objetivo final conseguir una mayor eficiencia en la medida y tratamiento del dolor en nuestro hospital.

El "Mapa del dolor" es una herramienta innovadora que facilita la gestión de las unidades de dolor agudo de bajo coste.

Puede acceder al vídeo del artículo con el siguiente código QR.

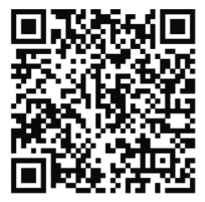

\section{CONFLICTO DE INTERESES}

Los autores declaran no tener ningún conflicto de intereses. 


\section{BIBLIOGRAFÍA}

1. Oficina de Planificación Sanitaria y Calidad. Desarrollo de la Estrategia Nacional en Seguridad del Paciente 2015-2020. Estrategia de Seguridad del Paciente del Sistema Nacional de Salud Período 2015-2020.[Internet] Madrid: Ministerio de Sanidad, Servicios Sociales e Igualdad. 2015 [Acceso el 14 de abril de 2015] Disponible en: http://www.seguridaddelpaciente.es/resources/documentos/2015/Estrategia\%20 Seguridad\%20del\%20Paciente\%202015-2020.pdf

2. Agencia de Calidad del Sistema Nacional de Salud. Plan de Calidad para el Sistema Nacional de Salud 2006. [Internet] Madrid: Ministerio de Sanidad y Consumo; Agencia de Calidad del Sistema Nacional de Salud; 2006. [Acceso el 19 de febrero de 2015]. Disponible en: http://www.msssi.gob. es/organizacion/sns/planCalidadSNS/pncalidad.htm

3. Palanca Sánchez I (Dir.), Puig Riera de Conías MM (Coord. Cient.), Elola Somoza J (Dir.), Bernal Sobrino JL (Comit. Redac.), Paniagua Caparrós JL (Comit. Redac.), Grupo de Expertos. Unidad de tratamiento de dolor: estándares y recomendaciones. Madrid: Ministerio de Sanidad, Política Social e lgualdad; 2011.

4. Miró J, Paredes S, Rull M, Queral R, Miralles R, Nieto R, et al. Pain in older adults: A prevalence study in the Mediterranean region of Catalonia. European Journal of Pain 2007;11(1):83-92. DOI: 10.1016/j.ejpain.2006.01.001.
5. De Andrés Gimeno MB, Salazar de la Guerra RM, Ferrer Arnedo C, Revuelta Zamorano M, Ayuso Murillo D, González Soria J. Una aproximación al benchmarking de indicadores de cuidados. Aprendiendo para mejorar la seguridad del paciente. Revista de calidad asistencial 2014;29(4):20129. DOI: 10.1016/j.cali.2014.04.002.

6. World Health Organization. Patient Safety. World Alliance for Patient Safety. Patient Safety.[Internet]. The Launch of the World Alliance for Patient Safety, Washington DC, USA 27 October 2004 [Accedido el 20 de febrero de 2015]. Disponible en: http://www.who.int/patientsafety/worldalliance/en/

7. Joint Commission on Acreditation of Healthcare Organizations. Pain assessment and management standards- Hospitals. Compprehensive Accreditation Manual for Hospitals. The oficial Handbook (CAMH). Oakbrook Terrace IL: Joint Commission on Acreditation of Healthcare Organizations, 2001.

8. Fillingim RB, King CD, Ribeiro-Dasilva MC, Rahim-Williams B, Riley JL. Sex, gender, and pain: a review of recent clinical and experimental findings. J Pain 2009;10(5):447-85. DOI: 10.1016/j.jpain.2008.12.001.

9. Rabanales J, Párraga I, López-Torres J, Andrés F, Navarro B. Tecnologías de la Información y las Telecomunicaciones: Telemedicina. Revista Clínica de Medicina de Familia 2011;4(1):42-8. 